\title{
Medical Risk Assessment in Patients Referred to Dental Clinics, Mashhad, Iran (2011-2012)
}

\author{
Amirchaghmaghi Maryam ${ }^{1}$, Pakfetrat Atessa ${ }^{1, *}$, Mosannen Mozafari Pegah $^{1}$, Shafiee Zahra ${ }^{2}$, \\ Ghalavani Hanieh $^{2}$, Aghasizadeh Davood ${ }^{3}$ and Khazaei Yeganeh ${ }^{4}$
}

\begin{abstract}
${ }^{I}$ Oral and Maxillofacial Diseases, Research Center, Department of Oral Medicine, Faculty of Dentistry, Mashhad, University of Medical Sciences, Mashhad, Iran; ${ }^{2}$ Mashhad University of Medical Sciences, Mashhad, Iran; ${ }^{3}$ Department of Prosthodontics, Faculty of Dentistry, Mashhad University of Medical Sciences, Mashhad, Iran; ${ }^{4}$ Faculty of Dentistry, Member of student Research Committee, Mashhad University of Medical Sciences, Mashhad, Iran
\end{abstract}

\begin{abstract}
Advances in medical and dental techniques have led to a growing aged population living with complex medical conditions. This study focuses on the detection of medically compromised dental patients by means of a validated patientadministered medical risk-related history questionnaire. Materials and Methods: We used the questionnaire EMRRH (European Risk Related Medical History) in order to study the prevalence as well as the risk assessment of past medical problems in a population who visited dental centers (dental university, dental offices and clinics) for treatment in Mashhad, Iran. Results: A total number of 1,188 patients were registered, 871 of whom had a medical history that was of some interest to us. From the population with medical problems which was $30.6 \%$ of the total (N=219), 26.7\% of the patients were classified as risk ASA I; $37.3 \%$ as ASA II, $16.9 \%$ as ASA III; and $19.1 \%$ as ASA IV. Among the diverse pathologies, the highest percentage was hypertension (11.6\%), followed by allergies to different drugs $(8.37 \%)$. Conclusion: It seems absolutely essential for dental practitioners to take a detailed medical history prior to any therapeutic procedure, as certain medical conditions, if unnoticed, will lead to unfavorable consequences and/or repercussions.
\end{abstract}

Keywords: ASA risk, dentistry, EMRRH, medical history.

\section{INTRODUCTION}

Advances in medical technology, wider access to medical facilities, and better socio-economic conditions have enabled people to live longer and better than ever before. The seemingly ever-increasing social greying, reported at an unprecedented scale over the past few years, can be translated to a rise in the number of patients receiving therapy, which, in turn, pose further medical complications at its wake [1-4].

So "apparently healthy" patients with serious chronic or past medical problems will need to have detailed medical histories taken before their dental treatment. Before commencing treatment, the dentist should make sure that these patients will not suffer any medical risk to their health [4-6].

The study carried out by Abraham-Inpijn et al. in 7 countries showed that there have been 120 deaths in dental surgeries over the last 10 years in Great Britain. In Holland, where 288 dentists had taken part in the study, 28 emergencies were reported the in one year, including sudden death as a result of myocardial infarction [1].Several studies also reported the frequency of systemic diseases among dental patients in various countries such as Saudi Arabia, Jordan and The West Indies with apparent disparities in figures and numbers. It is necessary to investigate the prevalence of systemic diseases among dental patients in various geographic places [7-10]. One essential aspect of the dentist's

*Address correspondence to this author at the Dental School, Vakil Abad BLVD, Mashhad, Postal code: 91735, Iran; Tel: 09155201889;

E-mail: pakfetrata@mums.ac.ir responsibility is to assess the medical history of patients before any treatment. Studies have shown that about half of the patients referred to dental schools had at least one medical complication [11, 12]. However, many dental practitioners assume that their patients are systemically healthy, with the expected result that medical history is often overlooked.

The aim of dental management is to make available safe and effective treatment, without precipitating a medical crisis. Accordingly, dental treatment might have to be altered to be consistent with the medical constraints of the patients, in order for providing their oral care, better and safer. Occasionally, consultation with a physician might be necessary [5].

There is a lack of data concerning the prevalence of medically compromised conditions in dental patients in Iran. Based on our research, only two studies have been carried out to evaluate the frequency of systemic diseases among patients who referred to endodontic offices and surgery departments (not all patients referred to dental school) in Iran [7, 11].

The search for an appropriate questionnaire has been the focus in several studies over the last few years. Dr. Abraham-Inpijn et al. from Holland has drawn up a questionnaire: the European Medical Risk Related History questionnaire (EMRRH), which was selected to be applied in the current research. Our main objective included:

1) To evaluate prevalence of systemic diseases in Mashhad dental centers.

2) Comparative risk assessment in patients referred to Mashhad dental centers based on gender, age, education, city or village and the type of disease. 


\section{METHODS AND MATERIALS}

This cross-sectional study was conducted over a span of eight months commencing in Nov 2011.Centres participating in the research include Mashhad dental school, private offices and clinics across the city of Mashhad, the state capital of Khorasan-Razavi province, north-east of Iran. The data were collected through personal interviews conducted by the same dentists who completed the patients' questionnaires.

The study population included 1,188 randomly selected patients, using a systematic sampling procedure from dental centres. Demographic information (age, gender, marital status, etc.) was collected. Meanwhile, the standard EMMRH questionnaire focusing on specific medical conditions including respiratory diseases, anemia, diabetes mellitus, hypertension, kidney problems, hepatic diseases, epilepsy, bleeding disorders and cardiovascular diseases was completed prior to treatment [1]. There were 23 main questions, each containing one or more detailed questions. All questions were given an ASA grade from I to IV. When there was a negative reply to the main question, the ASA risk was denoted as I. If the reply was affirmative, the risk would be designated as II, III or IV. The higher the degree of ASA was; the higher the level of risk for each patient was reported.

A positive reply was recorded as an ASA II risk, alerting the dentist to the presence of the disease. Each question was followed by 2 or more sub questions which indicated the increasing severity of the disease proportional to the score given. A positive answer to the first sub question gave rise to the score of ASA III and a subsequent positive reply to a sub question of the same principle question resulted in a score of ASA IV.

However, in this study 5 questions concerning the history of hemophilia, anemia, tobacco use, gastrointestinal diseases, and past blood transfusions were added to the questionnaire. When there were doubts concerning the patients' medical status, they were reexamined with medical consultation suggested if necessary. This study was approved by the ethical committee, Mashhad University of Medical Sciences, Iran.

\section{Statistical Analysis}

The analysis was carried out applying the SPSS software (SPSS for windows, version 11.5, Chicago, IL, USA). The standard median and deviation of age pertaining to the general population and individuals with and without medical histories were recorded. For the group of patients at medical risk, related histories related to each particular disease was taken. Subsequently the percentage of people suffering from these diseases in relation to the general population was calculated. The average age of the population with and without risk-related histories was compared using the Mann-Whitney test. The Pearson $\mathrm{X}^{2}$ test and t-test were chosen to assess the statistical significance in the prevalence of medical conditions among subgroups $(\mathrm{P}<0.05)$.

\section{RESULTS}

A total of 1,188 patients were studied (401,391 and 396 patients recruited from the Dental School of Mashhad, private offices, and dental clinics respectively). Their age was ranging from 8 to 92 years (64\% females, $36 \%$ male). Their mean age was $38.37 \pm 14.36$. The mean age of patients with medical risk-related histories was $39.28 \pm 15.65$ whereas patients without risk-related histories was recorded with a mean age of $35.77 \pm 13.48$, which was statistically significant $(\mathrm{p}<0.05)$ by Mann-Whitney test.

However, $97 \%(n=1,147)$ of patients were urban dwellers where as the remaining $3 \%(n=41)$ were originally from rural regions. The occupations also varied: housewives $(37.54 \%)$, students $(16.83 \%)$, businessmen $(14.6 \%)$, government employees $(12.2 \%)$, retired people $(5.05 \%)$, teachers $(3.03 \%)$, and unknown (10.69\%).

Out of a total of 1,188 patients, 871 patients were reported medically-compromised $(73.3 \%)$. Following categorization, most systemic diseases were seen to occur in individuals in their 30 s and 40 s while a few conditions were reported into extreme age brackets: $0-10$ and $80-90$ years of age.

The medically compromised group consisted of $52 \%$ $(n=601)$ female and 24\% $(n=270)$ male. The latter were diagnosed with considerable medical conditions with a statically higher proportion (Fig. 1).
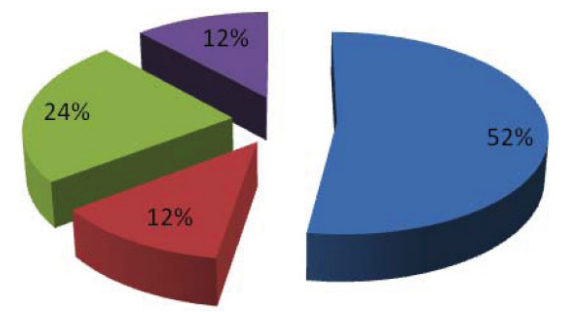

- Female patients

- Healthy Women

- Male patients

- Healthy Men

Fig. (1). Frequency of men and women with Medical problem.

In the present study the medical condition with the highest frequency regarded cardiovascular disease $(34.1 \%$; $\mathrm{n}=406)$, of these patients, $33.9 \%(\mathrm{n}=138)$ were suffering from hypertension.

The second most frequently medical condition was taking some sort of medication $(29 \%, n=345)$. At the bottom of the list were hemophilic patients $(0 \%)$.

As many as $29 \%$ of the total population were taking medication, with analgesics appearing on top of the list $(46.7 \% ; n=161)$, followed by drugs for hypertension $(29.2 \%$; $\mathrm{n}=101)$.

Gastrointestinal disturbance came third in the ranking $(18.01 \% ; n=214)$ after cardiovascular diseases and taking drugs in dental clinics and private dental offices, while the same rank was taken by anemia in Dental University Clinic.

The latter condition comprised a total of $17.1 \%$ of the study population whereas diabetes made up a mere $5.63 \%$, $83.58 \%$ of whom were insulin dependent (ASA II) whereas as many as $16.41 \%$ were not under sufficient control (ASAIII). Fig. (2) shows periodontitis and dental caries in diabetic patient referred to our dental clinic (Fig. 2).

From the patient with allergy, $1.2 \%(n=14)$ needed to be hospitalized or take medication. Of these, $0.2 \%(n=2)$ suffered a complication following prescriptions by the dentist. 


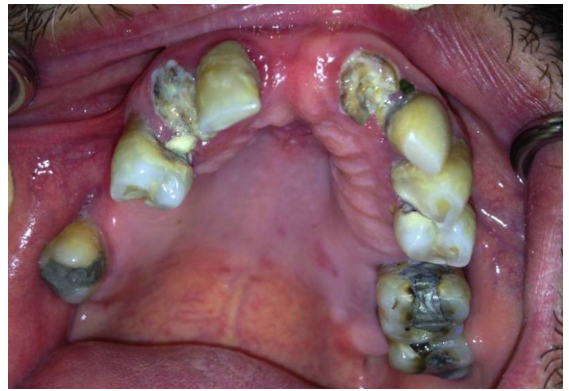

Fig. (2). periodontitis and dental caries in diabetic patient.

Data also indicated that frequencies differed in other special conditions: bleeding dyscrasias $4.6 \%(\mathrm{n}=55)$, respiratory problems $4.5 \%(n=54)$, thyroid diseases $4.5 \%(n=54)$,renal pathologies $3.8 \%(n=45)$, cancer and leukemia $0.75 \%(n=9)$ (Table 1). Fig. (3) shows peritoneal dialysis in patient referred to Dental clinic (Fig. 3).

Table 1. Frequency of medical condition.

\begin{tabular}{|c|c|c|}
\hline Percentage (\%) & Frequency & Medical condition \\
\hline 29 & 345 & Taking drug \\
\hline 18 & 214 & Gastrointestinal disorders \\
\hline 17.1 & 203 & Anemia \\
\hline 12 & 142 & antibiotic prophylaxis \\
\hline 11.6 & 138 & Hypertension \\
\hline 9.5 & 113 & chest pain \\
\hline 9.2 & 109 & Heart palpitation without exterior \\
\hline 2.2 & 26 & Heart attack \\
\hline 1.2 & 14 & Heart murmur \\
\hline 7.7 & 91 & Tobacco use \\
\hline 6.9 & 82 & History of allergy \\
\hline 6 & 71 & Received blood products \\
\hline 5.6 & 67 & Diabetes \\
\hline 5.6 & 67 & Bleeding \\
\hline 4.6 & 55 & Pulmonary disease \\
\hline 4.5 & 54 & Thyroid \\
\hline 3.8 & 45 & Renal disease \\
\hline 3.4 & 40 & asthma \\
\hline 2.3 & 28 & Liver diseases \\
\hline 0.75 & 9 & Cancer- leukemia \\
\hline 0.5 & 6 & Heart failure \\
\hline 0 & 0 & hemophilia \\
\hline
\end{tabular}


Table 2. Medical problems measured by the MRRH and their ASA risk scores.

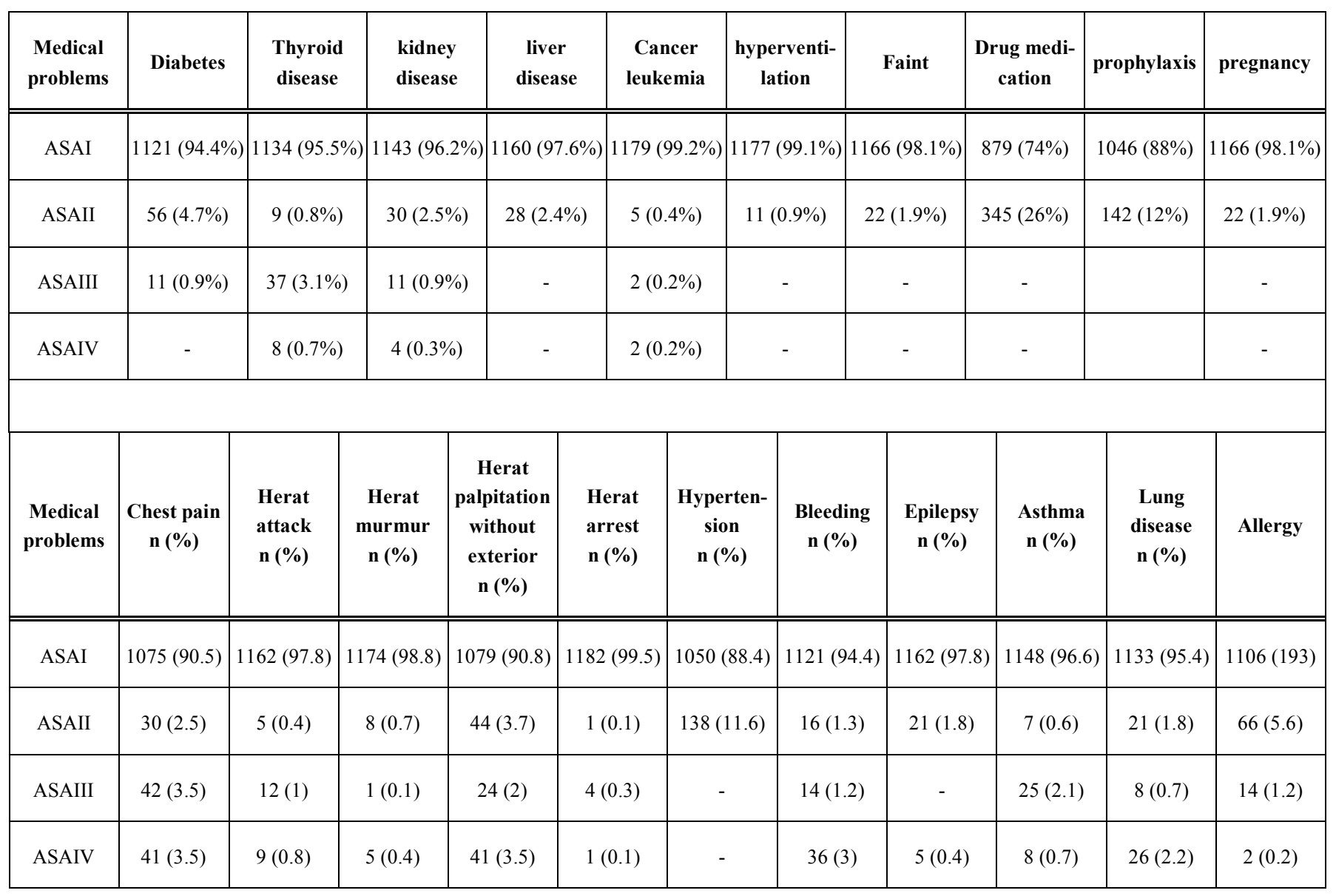

Gender predisposition ratio was 1.9 to 1 in this research (table), akin to Delavarian's [6] findings which included $38.2 \%$ male and $61.8 \%$ female, exceeding chandler's [4], reporting $40 \%$ and $60 \%$ for the same order of sexes and $46.1 \%$ men and $52.3 \%$ women in Smeet study (2). Radfar also came up with a ratio of 2 to 1 whereas Danuti reported 5 to1 $[13,14]$. This indication on the part of female population can be attributed to their greater health consciousness, from both oral and physical perspectives.

In our study, women comprised $69 \%$ of those diagnosed as "medically compromised", as opposed to men with will below half this figure (31\%).

The most common conditions included cardiovascular diseases, drug therapy, GI diseases and anemia, whereas Smeets' most prevalent list included allergy $(8.7 \%)$, heart diseases $(6.8 \%)$ hypertension $(4.4 \%)$ and respiratory diseases (3.2\%), indicating that COPD and allergy were rising along with aging [2].

Allergy was the most prevalent in Suomi, Saengsirinavin and Dhanuti findings $(8.3 \%)$. This condition came second following hypertension in Chandler's list [4, 14-16].

Al-Bayaty, Aggrawal reported hypertension and diabetes as common diseases $[10,17]$. Similar to our study, heart diseases were the most common condition in Belgium, Canada and Germany. By way of illustration, cardiac diseases (13\%) were the most frequent in the former nation followed by hy- pertension $(9 \%)$ and endocrine diseases $(8 \%)$. These conditions were also the most frequent in Germany (25\%), followed by drug- related allergies $(23 \%)$, anemia $(12 \%)$ and neurological diseases $(9 \%)[18,19]$.

Heart diseases were found in $34.1 \%, 14.9 \%$ and $15.6 \%$ of patients in the current study, chandlers' and Simmons' research respectively [4, 20-22]. This is while Glick from the Diagnostic Science Centre in New Jersey Dental School reported a mere $10 \%$ of prevalence for this morbid as well as fatal condition in USA [23].

Heart disease and hypertension were shown to have been closely interconnected (ASAII) as the latter was diagnosed in $34.9 \%$ and $13.8 \%$ of those with hypertension in our and chandler's study respectively [4].

Hypertension and cardiac disease were the most common medical problem encountered by Mesgarzadeh and Parrirok in order [7,11]. Given the somewhat similar prevalence of certain conditions namely cardiovascular, the pertinent management requires adequate knowledge and confidence on the part of the dentist.

The other factor to be taken into account is the large number of patients $(n=345)$, which comprised nearly $26 \%$ of the total population who are on some kind of medication. This is the consequence of the high level of medical background. It is known that all drugs have secondary effects, often interfering with dental treatment. 
In Chandler study, $24.1 \%$ of subjects took drugs [4], Similar to our findings which reported a high prevalence of drug prescription usage. The most common medication reported by our patients in this study was analgesics (13.65\%), followed by $8.50 \%$ of patients who were taking medications for hypertension. Similarly, Afolabi also came up with this group of drugs as the most commonly used [24]. In Chandler study anti-hypertension drugs and corticosteroids were the commonest whereas Radfar identified ant-histamines as the most prevalent $[4,13]$. The result of our study indicated that many patients were taking a variety of medications. The simultaneous use of variety of medications requires health care providers to be familiar with drugs and their interactions.

Respiratory diseases hovered around $8 \%$ of our population study while Chandler study claimed that $3.77 \%$ of this patients suffered from the conditions. This disease was as common as $3.14 \%$ and $3.47 \%$ in Japan Dental School and CDC study. In comparison ours were highest among other finding, urging special attention in this respect $[4,25,26]$.

In the United States $2-4 \%$ of the population is diabetic. The prevalence of diabetes is 6 times higher than 40 years ago [27]. Our research has shown that $5.6 \%$ of the total population was diabetic; $4.7 \%$ used insulin products and $0.9 \%$ was non-controlled diabetes. In the United States 90 $95 \%$ of diabetics have type II diabetes [28]. The incidence of type I diabetes has increased greatly in children and teenagers in last few years. The proportional distribution of type II diabetes rises from 8 cases per 100,000 at age 15 , to 163 cases per 100,000 at age 65 . This increase shows the effect of age on this disease [26].

There are very few reliable studies that show the prevalence by thyroid diseases. Studies carried out in Great Britain revealed 25-30 cases of hyperthyroidism per 10,000 women, and this disease is 10 times more frequent in women [29]. In our study, $0.7 \%$ of population study suffered from hyperthyroidism (ASAIV).

In Great Britain there are 3 cases per 1,000 women per year of hypothyroidism. It is estimated that there are 14 cases per 1,000 women and 1 per 1,000 men [29]. In our study $0.9 \%$ of the total population suffered from hypothyroidism (ASAIII). In the United States hypothyroidism was 5-6 times more frequent than hyperthyroidism [29], making statistics comparable with other studies.

As for the allergy-related conditions, a percentage of 6.9 of the total population study were reported with allergy, $17.1 \%$ of whom requiring hospitalization, whereas $2.4 \%$ experienced some kind of allergic reaction while receiving dental treatment. These figures were comparable to Chandler's reporting $8.37 \%, 1.5 \%$ and $3.6 \%$ for the mention conditions respectively. Despite the less prevalent nature of such conditions, the related risk valuation (ASAIII) gives rise to the importance of consideration in history taking. Allergy can cause multiform erythema, skin rashes, exanthemas and anaphylactic shock. Although patients with allergic problems are rare, but considering to risk evaluation (ASAIII), the importance of allergic background in taking history emphasized.

Having been based assessed as for as the prevalence of their conditions, the patients were subsequently classified based on the findings from their medical history. This riskrelated classification allowed the dentist to identify patients who can be treated with no risk at all while receiving treatments, as well as those whose treatment encompasses a greater degree of risk, however minimal. Similar studies using the same type of questionnaire have been carried out in other countries, where it was also shown that many patients had medical risk-related histories. For example, a study carried out in Holland with 29,424 patients having undergone dental surgery showed that $78 \%$ had an ASA risk I (with no medical risk histories); $12.7 \%$ ASA II; 5.7\% ASA risk III and $3.5 \%$ ASA risk IV [2].

The highest and lowest prevalence deleted in our study went for ASAII (37.3\%) and ASAIII (16.9\%) respectively. was the least common ASA grade in our study. A comparison was drawn by Abraham-Inpijn as for the prevalence of ASA degrees in 10 European countries in 1998 and 2008. They found that ASAI prevalence decreased from $78 \%$ to $57 \%$, implying that the percentage of medically compromised patients had increased [1]. The prevalence of various medical conditions affecting patients who had referred for dental treatment was also investigated by Abraham-Injipin based on European Medical Risk Related History [19, 25]. Another in Belgium involving a total number of 248 patients reported $57 \%$ of the subjects without any risk-related medical history (ASAI), whereas ASAI, II, IV affected 25\%, $11 \%$ and $7 \%$ in order $[19,25]$. By comparison, the record percentages pertaining to ASAII, III and IV classes belonged to those receiving drug therapies, patients with chest pain and cases of heart palpitation without exertion respectively. This is indicative of the fact that high-risk heart conditions are among the most common, thus meriting particular attention when taking medical history prior to treatment.

Given the high prevalence of the medical conditions in those referring for dental treatment, it seems of paramount importance for the dentist to take a through and precise medical history, preferably through a questionnaire which enables the practitioner to classify the patient instantaneously. Possible complications can thus be avoided.

\section{CONCLUSION}

The main conclusion of our study is a need for taking a complete clinical history of all patients, so avoiding any possible treatment that could put the patient's health or even his life, at risk. One of the types of clinical questionnaire that can be used is the EMRRH, due to its far-reaching and thorough questions. It is hoped that it will prove to be an invaluable tool for use in the dental practice. Dental schools may have to expand to include subjects such as the management of medically-compromised patients, pharmacology, and medical emergencies. Continuing education courses should emphasize these subjects as well. In dentistry, the curriculum may require modification toward a more medically-oriented dental education. Dental educators should reassess their materials and methods of education to ensure that dental practitioners are sufficiently equipped for meeting the population's requirements for dental care medical needs. Knowing about systemic diseases and medications will help dental practitioners to be aware of life-threatening situations that may occur during dental treatment. 


\section{CONFLICT OF INTEREST}

The authors confirm that this article content has no conflict of interest.

\section{ACKNOWLEDGEMENTS}

Declared none.

\section{REFERENCES}

[1] Abraham-Inpijn L1, Russell G, Abraham DA, et al. A patientadministered Medical Risk Related History questionnaire (EMRRH) for use in 10 European countries (multicenter trial). Oral Surg Oral Med Oral Pathol Oral Radiol Endod 2008; 105(5): 597605 .

[2] Smeets EC, Karin JM de Jong, Abraham-Inpijn L. Detecting the medically compromised patient in dentistry by means of the medical risk-related history; a survey of 29,424 dental patients in the Netherlands. Prev Med 1998; 27: 530-5.

[3] GoodchildJ H, Glick M. A different approach to medical risk assessment. Endod Topics 2003, 4: 1-8

[4] Chandler-Gutierrez L, Martinez-Sahuquillo A, Bullon-Fernandez P. Evaluation of medical risk in dental practice through using the EMRRH questionnaire. Med Oral 2004; 9: 309-20.

[5] Greenberg MS, Glick M. Burkets' Oral medicine diagnosis and treatment. $11^{\text {th }}$ ed. Hamilton: BC Decker 2008; pp. 1-15, 297-415, 509-45.

[6] Delavarian Z, Zavar S. An evaluation on patterns and causes of referring patients to oral diseases and diagnosis department of Mashhad dental faculty from September to December 2000. J Islam Dent Assoc Iran 2004; 16: 62-70.

[7] Parirokh M, Eghbal MJ, Ghoddusi J, Kakoei S, Haghdoost AA, Kakoei S. The frequency of Medically compromised patients in Endodontic offices in Iran. Iran Endodont J 2013; 8(2): 48-51.

[8] Almas K, Awartani FA. Prevalence of medically compromised patients referred for periodontal treatment to a teaching hospital in central Saudi Arabia. Saudi Med J 2003; 24(11): 1242-5.

[9] Khader YS, Alsaeed O, Burgan SZ, Amarin ZO. Prevalence of medical conditions among patients attending dental teaching clinics in northern Jordan. J Contemp Dent Pract 2007; 8(1): 60.

[10] Al-Bayaty H, Murti P, Naidu R, Matthews R, Simeon D. Medical problems among dental patients at the school of Dentistry, the University of the West Indies. J Dent Educ 2009; 73(12): 1408-14.

[11] Mesgarzadeh A, Mahmood-Hashemi H, Sharifi R, Hasheminasab M, Karimi A. A retrospective study of medically compromised patients referred to the Department of Oral and Maxillofacial Surgery, School of Dentistry of Tehran University of Medical Sciences, Iran. J Craniomax Res 2014; 1(1): 11-6.
[12] Andres PL, Comeau RL, Hatton M, Neiders ME. The nature and frequency of medical emergencies among patients in a dental school setting. J Dent Educ 2010; 74(4): 392-6.

[13] Radfar L, Suresh L. medical profile of a dental school patient population. J Dent Educ 2007; 71(5): 682-6.

[14] Dhanuthai K, Sappajatosok K, Bijaphala P, Kulutit S. Prealenccof medically compromised cnoditions in dental patients. Med Oral Patolroal Cir bucal 2009; 14(6): 287-91.

[15] Saengsirinavin C, Kraivaphan P, Phumara P. Survey of drug used and medical history among dental out-patient. J Am Dent Assoc 1990; 40: 68-74.

[16] Suomi JD, Horowitz HS, Barbano JP. Self-reported systemic conditions in an adult study population. Dent Res 1975; 54: 1092.

[17] Aggarwal A, Panat SR, Talukder S. Self-reported medical problems among dental patients in Western Uttar Pradesh, India. J Dent Educ 2011; 75(12): 1635-40.

[18] Jong de KJM, Abraham-Inpijn L. The validity of MRRH for dental patients in Belgium. Int Dent J 1997; 47: 16-20.

[19] Abraham-Inpijn L, Smeets EC, Russell JG, Abraham EA. Introductory notes regarding a European medical risk related history questionnaire (EMMRH) designed for use in dental practice. Br J Dent 1998; 185: 445-8.

[20] Simmons MS, Rhodous NL, Little JW. Screening dentists for risk factors associated with cardiovascular disease. Gen Dent 1994; 42: 440-6.

[21] Furberg CD, Psaty BM, ManolioTA. Prevalence of atrial fibrillation in elderly subjects: initial finding of the cardiovascular Health Study. JAMA1993; 269: 214-5.

[22] Waters BG. Providing dental treatment for patients with cardiovascular disease. Ont Dent 1995; 72(6): 24-6, 28-32.

[23] Glick M. Screening for traditional risk factors for cardiovascular disease: a review for oral health care providers. J Am Dent Assoc 2002; 133(3): 291-300.

[24] Afolabi AO, Akinmoladun VI, Adebose IJ, Elekwachi G. Self medication profile of dental patients in Ondo State, Nigeria. Niger J Med 2010; 19(1): 96-103.

[25] Akiyama K. Reviw of epidemiological studies on adult bronchial asthma in Japan. Nippon Kyobu Shikkan Gakkai Zasshi 1994; 32: s200-10. (abstract)

[26] Centers for disease control. Asthma--United States, 1982-1992. MMWR1995; 43: 952-3

[27] Little JW, Falace DA, Miller CS, Rhodus NL. Tratamiento Odontológico del Paciente Bajo Tratamiento Médico. Harcourt Brace 1998; 18: 387-409.

[28] Blackshear PJ. Diabetes mellitus. In: Kelly WA, Ed. Kelley's Textbook of Internal Medicine. $3^{\text {rd }}$ ed. Philadelphia: JB Lippincott; 1995; pp. 2022-30.

[29] Mark P, Vanderpump J. The epidemiology of thyroid disease. $\mathrm{Br}$ Med Bull 2011; 99(1): 39-51.

Received: March 31, 2015
(C) Maryam et al.; Licensee Bentham Open.

Revised: August 23, 2015

Accepted: September 23, 2015

This is an open access article licensed under the terms of the (https://creativecommons.org/licenses/by/4.0/legalcode), which permits unrestricted, noncommercial use, distribution and reproduction in any medium, provided the work is properly cited. 